\title{
MONODROMY, LIFTINGS OF HOLOMORPHIC MAPS, AND EXTENSIONS OF HOLOMORPHIC MOTIONS
}

\author{
YUNPING JIANG AND SUDEB MITRA \\ In memory of Professor Clifford J. Earle
}

\begin{abstract}
We study monodromy of holomorphic motions and show the equivalence of triviality of monodromy of holomorphic motions and extensions of holomorphic motions to continuous motions of the Riemann sphere. We also study liftings of holomorphic maps into certain Teichmüller spaces. We use this "lifting property" to prove that, under the condition of trivial monodromy, any holomorphic motion of a closed set in the Riemann sphere, over a hyperbolic Riemann surface, can be extended to a holomorphic motion of the sphere, over the same parameter space. We conclude that this extension can be done in a conformally natural way.
\end{abstract}

\section{INTRODUCTION}

Throughout this paper, we shall use the following notation: $\mathbb{C}$ for the complex plane, $\widehat{\mathbb{C}}=\mathbb{C} \cup\{\infty\}$ for the Riemann sphere, and $\Delta$ for the open unit disk $\{z \in \mathbb{C}$ : $|z|<1\}$.

The subject of holomorphic motions was introduced in the study of the dynamics of rational maps; see [19]. Since its inception, an important topic has been the question of extending holomorphic motions. The papers [5] and [26] contained partial results. Subsequently, Slodkowski showed that any holomorphic motion of a set in $\widehat{\mathbb{C}}$, over $\Delta$ as the parameter space, can be extended to a holomorphic motion of $\widehat{\mathbb{C}}$ over $\Delta$; see [25]; also refer to the papers [6] and [12]. The paper [1] used a group-equivariant version of Slodkowski's theorem to prove results in Teichmüller theory.

A natural question is to investigate holomorphic motions over more general parameter spaces. In Theorem 8.1 of [11, an explicit counterexample was given to show that Slodkowski's theorem does not, in general, hold when the parameter space is a domain in $\mathbb{C}^{2}$. In 15 , it was shown that Slodkowski's theorem does not hold for many higher-dimensional, simply connected parameter spaces; see also Proposition 5.4 in [16. This naturally leads us to consider the case when the parameter space is a hyperbolic Riemann surface. Theorem D of our paper answers this question.

Received by the editors August 15, 2017, and, in revised form, September 10, 2018.

2010 Mathematics Subject Classification. Primary 32G15; Secondary 30C62, 30F60, 30F99.

Key words and phrases. Teichmüller spaces, holomorphic maps, universal holomorphic motions.

The first author was partially supported by an NSF grant, a collaboration grant from the Simons Foundation (grant number 523341), and a grant from NSFC (grant number 11571122).

Both authors were partially supported by PSC-CUNY grants. 
A central topic is to study the question of obstructions to extending a holomorphic motion. More precisely, what are the analytic and topological obstructions to the extension of holomorphic motions? These two questions are intimately linked with Teichmüller spaces. The example in Proposition 5.4 of [16] studied the analytic obstruction. It is related to the non-existence of holomorphic sections for Teichmüller spaces; see the papers [8] and [9], and also [13. The example (Theorem 1) in [15] linked the question of extending holomorphic motions with Earle's result in 8. In our present paper, we study the question of topological obstructions. This is the essence of Theorem D.

The main purpose of our paper is to study necessary and sufficient topological conditions for extending holomorphic motions. We study monodromy of a holomorphic motion $\phi$ of a finite set $E$ in $\widehat{\mathbb{C}}$, defined over a connected complex Banach manifold $V$. We show the equivalence of the triviality of this monodromy and the extendability of $\phi$ to a continuous motion of $\widehat{\mathbb{C}}$ over $V$. We then show that, if $V$ is a hyperbolic Riemann surface, and $E$ is any set in $\widehat{\mathbb{C}}$, then, under the condition of trivial monodromy, any holomorphic motion of $E$ extends to a holomorphic motion of $\widehat{\mathbb{C}}$ over $V$.

The relationship between extending holomorphic motions and liftings of holomorphic maps into Teichmüller spaces of $\widehat{\mathbb{C}}$ with punctures, was first observed in Section 7 of the paper [5]. In that paper, the parameter space is the open unit disk. The main technique in our paper is to study the liftings of holomorphic maps from a hyperbolic Riemann surface into such Teichmüller spaces. Theorem B gives the general setting, and Theorem $\mathrm{C}$ is the key step in proving Theorem $\mathrm{D}$, which is the main result of this paper.

Our paper is organized as follows. In Section 1, we give all precise definitions and discuss the useful facts that will be necessary in the proofs of the main theorems of this paper. In Section 2, we give precise statements of the main theorems. In Sections $3,4,5$, and 6 , we prove the main theorems.

\section{Definitions And SOME FACts}

Definition 1.1. Let $V$ be a connected complex manifold with a basepoint $x_{0}$ and let $E$ be any subset of $\widehat{\mathbb{C}}$. A holomorphic motion of $E$ over $V$ is a map $\phi: V \times E \rightarrow \widehat{\mathbb{C}}$ that has the following three properties:

(1) $\phi\left(x_{0}, z\right)=z$ for all $z$ in $E$,

(2) the map $\phi(x, \cdot): E \rightarrow \widehat{\mathbb{C}}$ is injective for each $x$ in $V$, and

(3) the map $\phi(\cdot, z): V \rightarrow \widehat{\mathbb{C}}$ is holomorphic for each $z$ in $E$.

We say that $V$ is a parameter space of the holomorphic motion $\phi$. We will always assume that $\phi$ is a normalized holomorphic motion; i.e. 0,1 , and $\infty$ belong to $E$ and are fixed points of the map $\phi(x, \cdot)$ for every $x$ in $V$. It is sometimes useful to write $\phi(x, z)$ as $\phi_{x}(z)$.

If $E$ is a proper subset of $\widehat{E}$ and $\phi: V \times E \rightarrow \widehat{\mathbb{C}}, \widehat{\phi}: V \times \widehat{E} \rightarrow \widehat{\mathbb{C}}$ are two holomorphic motions, we say that $\widehat{\phi}$ extends $\phi$ if $\widehat{\phi}(x, z)=\phi(x, z)$ for all $(x, z)$ in $V \times E$.

Remark 1.2. Let $V$ and $W$ be connected complex manifolds with basepoints, and let $f$ be a basepoint preserving holomorphic map of $W$ into $V$. If $\phi$ is a holomorphic 
motion of $E$ over $V$ its pullback by $f$ is the holomorphic motion

$$
f^{*}(\phi)(x, z)=\phi(f(x), z) \quad \forall(x, z) \in W \times E,
$$

of $E$ over $W$.

Definition 1.3. Let $V$ be a connected complex manifold with a basepoint. Let $G$ be a group of Möbius transformations, let $E \subset \widehat{\mathbb{C}}$ be $G$-invariant, which means, $g(E)=E$ for each $g$ in $G$. A holomorphic motion $\phi: V \times E \rightarrow \widehat{\mathbb{C}}$ is $G$-equivariant if for any $x \in V, g \in G$ there is a Möbius transformation, denoted by $\theta_{x}(g)$, such that

$$
\phi(x, g(z))=\left(\theta_{x}(g)\right)(\phi(x, z))
$$

for all $z$ in $E$.

It is well known that if $\phi: V \times E \rightarrow \widehat{\mathbb{C}}$ is a holomorphic motion, where $V$ is a connected complex manifold with a basepoint $x_{0}$, then $\phi$ extends to a holomorphic motion of the closure $\bar{E}$, over $V$; see [19] and [3]. Hence, throughout this paper, we will assume that $E$ is a closed set in $\widehat{\mathbb{C}}$ (that contains the points 0,1 , and $\infty$ ).

Recall that a homeomorphism of $\widehat{\mathbb{C}}$ is called normalized if it fixes the points 0,1 , and $\infty$. The blanket assumption that $E$ is a closed set in $\widehat{\mathbb{C}}$ containing the points 0,1 , and $\infty$ holds.

Definition 1.4. Two normalized quasiconformal self-mappings $f$ and $g$ of $\widehat{\mathbb{C}}$ are said to be $E$-equivalent if and only if $f^{-1} \circ g$ is isotopic to the identity rel $E$. The Teichmüller space $T(E)$ is the set of all $E$-equivalence classes of normalized quasiconformal self-mappings of $\widehat{\mathbb{C}}$.

Let $M(\mathbb{C})$ be the open unit ball of the complex Banach space $L^{\infty}(\mathbb{C})$. Each $\mu$ in $M(\mathbb{C})$ is the Beltrami coefficient of a unique normalized quasiconformal homeomorphism $w^{\mu}$ of $\widehat{\mathbb{C}}$ onto itself. The basepoint of $M(\mathbb{C})$ is the zero function.

We define the quotient map

$$
P_{E}: M(\mathbb{C}) \rightarrow T(E)
$$

by setting $P_{E}(\mu)$ equal to the $E$-equivalence class of $w^{\mu}$, written as $\left[w^{\mu}\right]_{E}$. Clearly, $P_{E}$ maps the basepoint of $M(\mathbb{C})$ to the basepoint of $T(E)$.

In his doctoral dissertation [18, G. Lieb proved that $T(E)$ is a complex Banach manifold such that the projection map $P_{E}: M(\mathbb{C}) \rightarrow T(E)$ is a holomorphic split submersion; see [1] for the details.

Remark 1.5. Let $E$ be a finite set. Its complement $\Omega=\widehat{\mathbb{C}} \backslash E$ is the Riemann sphere with punctures at the points of $E$. Then, $T(E)$ is biholomorphic to the classical Teichmüller space Teich $(\Omega)$; see Example 3.1 in [20] for the proof. This canonical identification will be very important in our paper. (The reader is referred to [14] or 23] for standard facts in Teichmüller theory.)

Proposition 1.6. There is a continuous basepoint preserving map s from $T(E)$ to $M(\mathbb{C})$ such that $P_{E} \circ s$ is the identity map on $T(E)$.

See [1], 16] for all details.

Definition 1.7. The map $s$ from $T(E)$ to $M(\mathbb{C})$ is called the Douady-Earle section of $P_{E}$ for the Teichmüller space $T(E)$. 
Remark 1.8. When $E$ is finite, $T(E)$ is canonically identified with the classical Teichmüller space Teich $(\widehat{\mathbb{C}} \backslash E)$, and hence $s$ is the continuous section studied in Lemma 5 in 7 .

Definition 1.9. The universal holomorphic motion $\Psi_{E}: T(E) \times E \rightarrow \widehat{\mathbb{C}}$ is defined as follows:

$$
\Psi_{E}\left(P_{E}(\mu), z\right)=w^{\mu}(z) \text { for } \mu \in M(\mathbb{C}) \text { and } z \in E .
$$

It is clear from the definition of $P_{E}$ that the map $\Psi_{E}$ is well-defined. It is a holomorphic motion because $P_{E}$ is a holomorphic split submersion and $\mu \mapsto w^{\mu}(z)$ is a holomorphic map from $M(\mathbb{C})$ to $\widehat{\mathbb{C}}$ for every fixed $z$ in $\widehat{\mathbb{C}}$, by Theorem 11 in $[2]$.

This holomorphic motion is "universal" in the following sense.

Theorem 1.10. Let $\phi: V \times E \rightarrow \widehat{\mathbb{C}}$ be a holomorphic motion. If $V$ is a simply connected complex Banach manifold with a basepoint, there is a unique basepoint preserving holomorphic map $f: V \rightarrow T(E)$ such that $f^{*}\left(\Psi_{E}\right)=\phi$.

For a proof see Section 14 in [20].

Remark 1.11. Let $\phi: V \times E \rightarrow \widehat{\mathbb{C}}$ be a holomorphic motion, where $V$ is a connected complex Banach manifold with a basepoint $x_{0}$. Suppose there exists a basepoint preserving holomorphic map $f: V \rightarrow T(E)$ such that $f^{*}\left(\Psi_{E}\right)=\phi$. Let $\widetilde{f}: V \rightarrow$ $M(\mathbb{C})$, where $\tilde{f}=s \circ f$. By Proposition 1.6, $\tilde{f}$ is a basepoint preserving continuous map. Then, for all $(x, z) \in V \times E$, we have

$$
\phi(x, z)=\Psi_{E}(f(x), z)=\Psi_{E}\left(P_{E}(s(f(x))), z\right)=w^{s(f(x))}(z)=w^{\widetilde{f}(x)}(z) .
$$

Definition 1.12. Let $W$ be a path-connected Hausdorff space with a basepoint $x_{0}$. A (normalized) continuous motion of $\widehat{\mathbb{C}}$ over $W$ is a continuous map $\phi: W \times \widehat{\mathbb{C}} \rightarrow \widehat{\mathbb{C}}$ such that:

(a) $\phi\left(x_{0}, z\right)=z$ for all $z \in \widehat{\mathbb{C}}$ and

(b) for each $x$ in $W$, the map $\phi(x, \cdot):=\phi_{x}(\cdot)$ is a homeomorphism of $\widehat{\mathbb{C}}$ onto itself that fixes the points 0,1 , and $\infty$.

In [21] the following was shown.

Theorem 1.13. Let $\phi: V \times E \rightarrow \widehat{\mathbb{C}}$ be a holomorphic motion, where $V$ is a connected complex Banach manifold with a basepoint $x_{0}$. Then the following are equivalent:

(i) There is a continuous motion $\widetilde{\phi}: V \times \widehat{\mathbb{C}} \rightarrow \widehat{\mathbb{C}}$ that extends $\phi$.

(ii) There exists a basepoint preserving holomorphic map $F: V \rightarrow T(E)$ such that $F^{*}\left(\Psi_{E}\right)=\phi$.

The following corollary will be useful in this paper; see [21].

Corollary 1.14. If the holomorphic motion $\phi$ can be extended to a continuous motion $\widetilde{\phi}$, then $\widetilde{\phi}$ can be chosen so that:

(i) the map $\widetilde{\phi}_{x}: \widehat{\mathbb{C}} \rightarrow \widehat{\mathbb{C}}$ is quasiconformal for each $x$ in $V$ and

(ii) its Beltrami coefficient $\mu_{x}$ is a continuous function of $x$.

Let $w$ be a normalized quasiconformal self-mapping of $\widehat{\mathbb{C}}$, and let $\widetilde{E}=w(E)$. By definition, the allowable map $g$ from $T(\widetilde{E})$ to $T(E)$ maps the $\widetilde{E}$-equivalence class of $f$ to the $E$-equivalence class of $f \circ w$ for every normalized quasiconformal self-mapping $f$ of $\widehat{\mathbb{C}}$. 
Lemma 1.15. The allowable map $g: T(\widetilde{E}) \rightarrow T(E)$ is biholomorphic. If $\mu$ is the Beltrami coefficient of $w$, then $g$ maps the basepoint of $T(\widetilde{E})$ to the point $P_{E}(\mu)$ in $T(E)$.

See $\S 7.12$ in [11] or $\S 6.4$ in [20] for a complete proof. The following lemma will be useful in our paper.

Lemma 1.16. Let $B$ be a path-connected topological space. Let $f$ and $g$ be two continuous maps from $B$ to $T(E)$, satisfying:

(i) $\Psi_{E}(f(t), z)=\Psi_{E}(g(t), z)$ for all $z$ in $E$ and

(ii) $f\left(t_{0}\right)=g\left(t_{0}\right)$ for some $t_{0}$.

Then, $f(t)=g(t)$ for all $t$ in $B$.

See $\S 12$ in 20 for the proof.

If $f(t)=\left[w^{\mu}\right]_{E}$ and $g(t)=\left[w^{\nu}\right]_{E}$, condition (i) of the lemma means that $w^{\mu}(z)=$ $w^{\nu}(z)$ for all $z$ in $E$.

If $E$ is a subset of the closed set $\widehat{E}$ and $\mu$ is in $M(\mathbb{C})$, then the $\widehat{E}$-equivalence class of $w^{\mu}$ is contained in the $E$-equivalence class of $w^{\mu}$. Therefore, there is a well-defined "forgetful map"

$$
p_{\widehat{E}, E}: T(\widehat{E}) \mapsto T(E)
$$

such that $P_{E}=p_{\widehat{E}, E} \circ P_{\widehat{E}}$. It is easy to see that this is a basepoint preserving holomorphic split submersion.

The following is a consequence of Lemma 1.16. Here, $\Psi_{E}$ is the universal holomorphic motion of $E$ and $\Psi_{\widehat{E}}$ is the universal holomorphic motion of $\widehat{E}$.

Proposition 1.17. Let $V$ be a connected complex Banach manifold with basepoint, and let $f$ and $g$ be basepoint preserving holomorphic maps from $V$ into $T(E)$ and $T(\widehat{E})$, respectively. Then $p_{\widehat{E}, E} \circ g=f$ if and only if $g^{*}\left(\Psi_{\widehat{E}}\right)$ extends $f^{*}\left(\Psi_{E}\right)$.

See $\S 13$ in 20] for the proof. We say that the holomorphic map $g$ lifts the holomorphic map $f$.

We now discuss the concept of monodromy of a holomorphic motion. We closely follow the discussion in $\S 2$ in [4]. Let $\phi: V \times E \rightarrow \widehat{\mathbb{C}}$ be a holomorphic motion, where $V$ is a connected complex Banach manifold with a basepoint $x_{0}$. Let $\pi: \widetilde{V} \rightarrow V$ be a holomorphic universal covering, with the group of deck transformations $\Gamma$. We choose a point $\widetilde{x}_{0}$ in $\widetilde{V}$ such that $\pi\left(\widetilde{x}_{0}\right)=x_{0}$. Let $\pi_{1}\left(V, x_{0}\right)$ denote the fundamental group of $V$ with basepoint $x_{0}$.

Let $\Phi=\pi^{*}(\phi)$. Then, $\Phi: \widetilde{V} \times E \rightarrow \widehat{\mathbb{C}}$ is a holomorphic motion of $E$ over $\widetilde{V}$ with $\widetilde{x}_{0}$ as the basepoint. By Remark 1.11, there exists a basepoint preserving continuous map $\widetilde{f}: \widetilde{V} \rightarrow M(\mathbb{C})$ such that

$$
\Phi(x, z)=w^{\widetilde{f}(x)}(z)
$$

for each $x \in \widetilde{V}$ and each $z \in E$.

For each $z \in E$ and for each $\gamma \in \Gamma$, we have

$$
w^{\widetilde{f} \circ \gamma\left(\widetilde{x}_{0}\right)}(z)=\Phi\left(\gamma\left(\widetilde{x}_{0}\right), z\right)=\phi\left(\pi \circ \gamma\left(\widetilde{x}_{0}\right), z\right)=\phi\left(x_{0}, z\right)=z .
$$

Therefore, $w^{\widetilde{f} \circ \gamma\left(\widetilde{x}_{0}\right)}$ keeps every point of $E$ fixed.

Lemma 1.18. The homotopy class of $w^{\widetilde{f} \circ \gamma\left(\widetilde{x}_{0}\right)}$ relative to $E$ does not depend on the choice of the continuous map $\widetilde{f}$. 
See Lemma 2.12 in [4].

We now assume that $E$ is a finite set containing $n$ points, where $n \geq 4$; as usual, 0,1 , and $\infty$ are in $E$. Let $\phi: V \times E \rightarrow \widehat{\mathbb{C}}$ be a holomorphic motion. The map $w^{\widetilde{f} \circ \gamma\left(\widetilde{x}_{0}\right)}$ is a quasiconformal self-map of the hyperbolic Riemann surface $X_{E}:=\widehat{\mathbb{C}} \backslash E$. Therefore, it represents a mapping class of $X_{E}$, and by Lemma 1.18. we have a homomorphism $\rho_{\phi}: \pi_{1}\left(V, x_{0}\right) \rightarrow \operatorname{Mod}(0, n)$ given by

$$
\rho_{\phi}(c)=\left[w^{\tilde{f} \circ \gamma\left(\widetilde{x}_{0}\right)}\right],
$$

where $\operatorname{Mod}(0, n)$ is the mapping class group of the $n$-times punctured sphere, $\gamma \in \Gamma$ is the element corresponding to $c \in \pi_{1}\left(V, x_{0}\right)$, and $[w]$ denotes the mapping class group of $X_{E}$ for $w$.

Definition 1.19. We call the homomorphism $\rho_{\phi}$ the monodromy of the holomorphic motion $\phi$ of the finite set $E$. The monodromy is called trivial if it maps every element of $\pi_{1}\left(V, x_{0}\right)$ to the identity of $\operatorname{Mod}(0, n)$.

\section{Statements of the MAIN TheOREMS}

In this section, we give the precise statements of the main theorems of our paper.

Theorem A. Let $\phi: V \times E \rightarrow \widehat{\mathbb{C}}$ be a holomorphic motion of a finite set $E$, containing the points 0,1 , and $\infty$, where $V$ is a connected complex Banach manifold with basepoint $x_{0}$. The following are equivalent:

(i) There exists a continuous motion $\widetilde{\phi}: V \times \widehat{\mathbb{C}} \rightarrow \widehat{\mathbb{C}}$, such that $\widetilde{\phi}$ extends $\phi$.

(ii) The monodromy $\rho_{\phi}$ is trivial.

In the next theorem, let $\widehat{E}=E \cup\{\zeta\}$, where, $E$ is a finite set containing 0 , 1 , and $\infty$, and $\zeta \in \mathbb{C} \backslash E$. Let $V$ be a connected complex Banach manifold with basepoint $x_{0}$.

Theorem B. Suppose every holomorphic map from $V$ into $T(E)$ lifts to a holomorphic map from $V$ into $T(\widehat{E})$. Then, if $\phi: V \times E \rightarrow \widehat{\mathbb{C}}$ is a holomorphic motion that has trivial monodromy, there exists a holomorphic motion $\widehat{\phi}: V \times \widehat{E} \rightarrow \widehat{\mathbb{C}}$ such that $\widehat{\phi}$ extends $\phi$ and also has trivial monodromy.

In the next two theorems, $X$ is a hyperbolic Riemann surface with a basepoint $x_{0}$, and $E$ is a closed set in $\widehat{\mathbb{C}}$ containing the points 0,1 , and $\infty$.

Let $E_{n}=\left\{0,1, \infty, \xi_{1}, \cdots, \xi_{n}\right\}$, where $n \geq 1$, and $E_{n+1}=E_{n} \cup\left\{\xi_{n+1}\right\}$, where $\xi_{n+1} \in \widehat{\mathbb{C}} \backslash E_{n}$. Let $p: T\left(E_{n+1}\right) \rightarrow T\left(E_{n}\right)$ denote the forgetful map in (1.2). Let $\phi_{n}: X \times E_{n} \rightarrow \widehat{\mathbb{C}}$ be a holomorphic motion that has trivial monodromy. We will see in the proof of Theorem $\mathrm{A}$ that there exists a unique basepoint preserving holomorphic map $f_{n}: X \rightarrow T\left(E_{n}\right)$ such that $f_{n}^{*}\left(\Psi_{E_{n}}\right)=\phi_{n}$. The following theorem is a key result in our paper.

Theorem C. Let $\phi_{n}: X \times E_{n} \rightarrow \widehat{\mathbb{C}}$ be a holomorphic motion. If the monodromy of $\phi_{n}$ is trivial, there exists a basepoint preserving holomorphic map $f_{n+1}: X \rightarrow$ $T\left(E_{n+1}\right)$ such that $p \circ f_{n+1}=f_{n}$.

The following corollary is an immediate consequence. Here $\Psi_{E_{n+1}}: T\left(E_{n+1}\right) \times$ $E_{n+1} \rightarrow \widehat{\mathbb{C}}$ is the universal holomorphic motion of $E_{n+1}$.

Corollary 2.1. Let $\phi_{n+1}:=f_{n+1}^{*}\left(\Psi_{E_{n+1}}\right)$. Then $\phi_{n+1}$ extends $\phi_{n}$ and has trivial monodromy. 
Theorem D. Let $\phi: X \times E \rightarrow \widehat{\mathbb{C}}$ be a holomorphic motion such that $\phi$ restricted to $X \times E^{\prime}$ has trivial monodromy, or extends to a continuous motion of $\widehat{\mathbb{C}}$ (over $X)$, where $E^{\prime}$ is any finite subset of $E$, containing the points 0,1 , and $\infty$. Then, there exists a holomorphic motion $\widehat{\phi}: X \times \widehat{\mathbb{C}} \rightarrow \widehat{\mathbb{C}}$ such that $\widehat{\phi}$ extends $\phi$.

Remark 2.2. H. Shiga 24 has recently announced a completely different approach to a part of this theorem. Our methods are totally independent and more direct. The crucial point in our approach is the lifting property as given in Theorem C.

\section{Proof of Theorem A}

Let $\pi: \widetilde{V} \rightarrow V$ be a holomorphic universal covering with the group $\Gamma$ of deck transformations, so that, $V=\widetilde{V} / \Gamma$, and $\pi\left(\widetilde{x}_{0}\right)=x_{0}$.

Suppose $\phi$ can be extended to a continuous motion $\widetilde{\phi}$ of $\widehat{\mathbb{C}}$ over $V$. Then, by Corollary 1.14 there exists a continuous map $f: V \rightarrow M(\mathbb{C})$ such that $\widetilde{\phi}(x, z)=$ $w^{f(x)}(z)$ for all $(x, z) \in V \times \widehat{\mathbb{C}}$. Let $\tilde{f}=f \circ \pi$. Then, for any $c \in \pi_{1}\left(V, x_{0}\right)$ with corresponding $\gamma \in \Gamma$, we have

$$
\rho_{\phi}(c)=\left[w^{\widetilde{f} \circ \gamma\left(\widetilde{x}_{0}\right)}\right]=\left[w^{f \circ \pi \circ \gamma\left(\widetilde{x}_{0}\right)}\right]=\left[w^{f\left(x_{0}\right)}\right]=[I d] .
$$

This shows that the monodromy $\rho_{\phi}$ is trivial.

Let $\phi: V \times E \rightarrow \widehat{\mathbb{C}}$ be a holomorphic motion with trivial monodromy. Let $\phi_{\widetilde{V}}:=\pi^{*}(\phi)$ be the holomorphic motion of $E$ over $\widetilde{V}$. By Theorem 1.10, there exists a unique basepoint preserving holomorphic map $f_{\widetilde{V}}: \widetilde{V} \rightarrow T(E)$, such that $\phi_{\widetilde{V}}=f_{\widetilde{V}}^{*}\left(\Psi_{E}\right)$. For any element $\gamma \in \Gamma$, we also have $f_{\widetilde{V}} \circ \gamma: \widetilde{V} \rightarrow T(E)$. Note that

$$
\begin{gathered}
\left(f_{\widetilde{V}} \circ \gamma\right)^{*}\left(\Psi_{E}\right)(x, z)=\Psi_{E}\left(\left(f_{\widetilde{V}} \circ \gamma\right) x, z\right)=\phi_{\widetilde{V}}(\gamma(x), z)=\phi(\pi(\gamma(x)), z) \\
=\phi(\pi(x), z)=\phi_{\widetilde{V}}(x, z)=\left(f_{\widetilde{V}}\right)^{*}\left(\Psi_{E}\right)(x, z) .
\end{gathered}
$$

By the triviality of the monodromy, we have $f_{\widetilde{V}} \circ \gamma\left(x_{0}\right)=f_{\widetilde{V}}\left(x_{0}\right)$ for all $\gamma \in \Gamma$. Lemma 1.16 implies that $f_{\widetilde{V}} \circ \gamma=f_{\widetilde{V}}$ for all $\gamma \in \Gamma$. Thus, $f_{\widetilde{V}}$ defines a unique basepoint preserving holomorphic map $f: V \rightarrow T(E)$ such that $\phi=f^{*}\left(\Psi_{E}\right)$. It then follows from Theorem 1.13 that there exists a continuous motion of $\widehat{\mathbb{C}}$ over $V$ that extends $\phi$. This completes the proof.

\section{Proof of Theorem B}

Let $\phi: V \times E \rightarrow \widehat{\mathbb{C}}$ be a holomorphic motion such that it has trivial monodromy. By the proof of Theorem A, there exists a basepoint preserving holomorphic map $f: V \rightarrow T(E)$ such that $\phi=f^{*}\left(\Psi_{E}\right)$. Let $p: T(\widehat{E}) \rightarrow T(E)$ denote the forgetful map defined in (1.2). By hypothesis, there exists a basepoint preserving holomorphic map $\widehat{f}: V \rightarrow T(\widehat{E})$ such that $p \circ \widehat{f}=f$. Let $\widehat{\phi}:=\widehat{f}^{*}\left(\Psi_{\widehat{E}}\right)$. By Proposition 1.17, $\widehat{\phi}$ extends $\phi$. Note that, for $x \in V$, and $z \in \widehat{E}$, we have $\widehat{\phi}(x, z)=w^{\alpha(x)}(z)$, where $\alpha=s_{\widehat{E}} \circ \widehat{f}$ and $s_{\widehat{E}}$ is the continuous section of the projection $P_{\widehat{E}}: M(\mathbb{C}) \rightarrow T(\widehat{E})$; see Remark 1.8

Let $\pi: \widetilde{V} \rightarrow V$ be the holomorphic universal cover, and $\pi\left(\widetilde{x}_{0}\right)=x_{0}$. Then, $\pi^{*}(\widehat{\phi}): \widetilde{V} \times \widehat{E} \rightarrow \widehat{\mathbb{C}}$ is a holomorphic motion. Since $\widetilde{V}$ is simply connected, there exists a basepoint preserving continuous map $\beta: \widetilde{V} \rightarrow M(\mathbb{C})$ such that $\beta^{*}\left(\Psi_{\widehat{E}}\right)=$ 
$\pi^{*}(\widehat{\phi})$ (see Remark 1.11). That implies $\pi^{*}(\widehat{\phi})(x, z)=w^{\beta(x)}(z)$. Recall that the monodromy $\rho: \pi_{1}(V) \rightarrow \operatorname{Mod}(0, n+1)$ is defined by

$$
\rho(c)=\left[w^{\beta \circ \gamma\left(x_{0}\right)}\right]
$$

for any $c \in \pi_{1}\left(V, x_{0}\right)$ with the corresponding $\gamma \in \Gamma$. Furthermore, it is independent of the choice of $\beta$. In particular, if we choose $\beta=\alpha \circ \pi$, we see that

$$
\rho(c)=\left[w^{\alpha \circ \pi \circ \gamma\left(x_{0}\right)}\right]=\left[w^{\alpha\left(x_{0}\right)}\right]=[I d] .
$$

Note that $\pi \circ \gamma\left(x_{0}\right)=x_{0}$. This implies that $\rho$ is trivial.

\section{Proofs of Theorem C and Corollary 2.1}

We recall the following result, due to $\mathrm{S}$. Nag, that will be fundamental in our paper; see [22].

Theorem 5.1. Given $n>0$, choose a point $\left(\zeta_{1}, \cdots, \zeta_{n}\right)$ in the domain

$$
Y_{n}=\left\{\left(z_{1}, \cdots, z_{n}\right) \in \mathbb{C}^{n}: z_{i} \neq z_{j} \text { for } i \neq j \text { and } z_{i} \neq 0,1 \text { for all } i=1, \cdots, n\right\}
$$

and let $E_{n}=\left\{0,1, \infty, \zeta_{1}, \cdots, \zeta_{n}\right\}$. Then, the map $p_{n}: T\left(E_{n}\right) \rightarrow Y_{n}$ defined by

$$
p_{n}\left(\left[w^{\mu}\right]_{E_{n}}\right)=\left(w^{\mu}\left(\zeta_{1}\right), \cdots, w^{\mu}\left(\zeta_{n}\right)\right) \text { for all } \mu \in M(\mathbb{C})
$$

is a holomorphic universal covering.

Let $E_{n}=\left\{0,1, \infty, \zeta_{1}, \cdots, \zeta_{n}\right\}$ and $E_{n+1}=E_{n} \cup\left\{\zeta_{n+1}\right\}$, where $\zeta_{n+1} \in \widehat{\mathbb{C}} \backslash E_{n}$. We have also a holomorphic universal covering $p_{n+1}: T\left(E_{n+1}\right) \rightarrow Y_{n+1}$, where $Y_{n+1}=\left\{\left(z_{1}, \cdots, z_{n+1}\right) \in \mathbb{C}^{n+1}: z_{i} \neq z_{j}\right.$ for $i \neq j$ and $z_{i} \neq 0,1$ for all $\left.i=1, \cdots, n+1\right\}$.

Let $p: T\left(E_{n+1}\right) \rightarrow T\left(E_{n}\right)$ denote the forgetful map defined in (1.2).

We need some preliminaries. The reader is referred to Lemmas 3.1-3.5 in [17] for all details. Let $\mathcal{C}(\mathbb{C})$ denote the complex Banach space of bounded, continuous functions $\phi$ on $\mathbb{C}$ with the norm

$$
\|\phi\|=\sup _{z \in \mathbb{C}}|\phi(z)| .
$$

In $\S 3$ of [17] we construct a continuous compact operator

$$
\mathcal{K}: \mathcal{C}(\mathbb{C}) \rightarrow \mathcal{C}(\mathbb{C})
$$

By Lemma 3.2 in [17, there exists a constant $C_{3}>0$ such that

$$
\|\mathcal{K}\| \leq C_{3} \text { for all } f \in \mathcal{C}(\mathbb{C}) .
$$

For $\zeta_{n+1}$, let

$$
\mathcal{B}=\left\{f \in \mathcal{C}(\mathbb{C}):\|f\| \leq\left|\zeta_{n+1}\right|+C_{3}\right\}
$$

It is a bounded convex subset in $\mathcal{C}(\mathbb{C})$. The continuous compact operator $\zeta_{n+1}+\mathcal{K}$ maps $\mathcal{B}$ into itself. By Schauder fixed point theorem (see Theorem $2 \mathrm{~A}$ on page 56 of [27; also page 557 of [17]), $\zeta_{n+1}+\mathcal{K}$ has a fixed point in $\mathcal{B}$. This says that we can find a $g_{n+1} \in \mathcal{B}$ such that

$$
g_{n+1}(z)=\zeta_{n+1}+\mathcal{K} g_{n+1}(z) \text { for all } z \in \mathbb{C} .
$$

The reader is referred to $\S 3$ of [17] for all details; especially page 557 of that paper. By Lemma 3.5 of [17, the solution $g_{n+1}(z)$ is the unique fixed point of the operator $\zeta_{n+1}+\mathcal{K}$.

Suppose $\phi_{n}: X \times E_{n} \rightarrow \widehat{\mathbb{C}}$ is a holomorphic motion that has trivial monodromy. By Theorem A, it can be extended to a continuous motion $\widetilde{\phi}: X \times \widehat{\mathbb{C}} \rightarrow \widehat{\mathbb{C}}$. By 
Theorem 1.13 there exists a basepoint preserving holomorphic map $f_{n}: X \rightarrow$ $T\left(E_{n}\right)$ such that $f_{n}^{*}\left(\Psi_{E}\right)=\phi_{n}$.

Proof of Theorem C. Let

$$
p_{n}: T\left(E_{n}\right) \rightarrow Y_{n} \quad \text { and } \quad p_{n+1}: T\left(E_{n+1}\right) \rightarrow Y_{n+1}
$$

be the two holomorphic coverings in Theorem 5.1 .

Since $X$ is a hyperbolic Riemann surface, its universal covering space is the open unit disk $\Delta$. Let $\pi: \Delta \rightarrow X$ be the holomorphic universal cover. Let $\Gamma$ be the group of deck transformations such that $X=\Delta / \Gamma$.

Define the holomorphic map

$$
f_{\Delta, n}=f_{n} \circ \pi: \Delta \rightarrow T\left(E_{n}\right)
$$

and for any given $\gamma \in \Gamma$, consider the holomorphic map

$$
f_{\Delta, n} \circ \gamma: \Delta \rightarrow T\left(E_{n}\right)
$$

So we have two holomorphic maps

$$
F_{n}=p_{n} \circ f_{\Delta, n}: \Delta \rightarrow Y_{n} \quad \text { and } \quad F_{n} \circ \gamma=p_{n} \circ\left(f_{\Delta, n} \circ \gamma\right): \Delta \rightarrow Y_{n} .
$$

Let us write

$$
F_{n}=\left(h_{1}, \cdots, h_{n}\right) \quad \text { and } \quad F_{n} \circ \gamma=\left(h_{1} \circ \gamma, \cdots, h_{n} \circ \gamma\right) .
$$

Each $h_{i}$ (as well as $\left.h_{i} \circ \gamma\right)$ is holomorphic in $\Delta$. In $\S 3$ of [10], we constructed a map $g_{i}$ (as well as a map $g_{i} \circ \gamma$ ) by using $h_{i}$ (as well as $h_{i} \circ \gamma$ ) which is holomorphic outside $\Delta$ and continuous on $\mathbb{C}$. By using $g_{i}$ for all $1 \leq i \leq n$, we constructed a continuous compact operator

$$
\mathcal{K}=\mathcal{K}\left(F_{n}\right): \mathcal{C}(\mathbb{C}) \rightarrow \mathcal{C}(\mathbb{C})
$$

in $\S 3$ of [10]. Similarly, by using $g_{i} \circ \gamma$ for all $1 \leq i \leq n$, we have a continuous compact operator

$$
\mathcal{K}_{\gamma}=\mathcal{K}\left(F_{n} \circ \gamma\right): \mathcal{C}(\mathbb{C}) \rightarrow \mathcal{C}(\mathbb{C})
$$

The main point in $\S 3$ of [10] is that we can find the unique fixed point $g_{n+1}$ for $\xi_{n+1}+\mathcal{K}$ and the unique fixed point $g_{n+1, \gamma}$ for $\xi_{n+1}+\mathcal{K}_{\gamma}$. That is,

$$
g_{n+1}(z)=\xi_{n+1}+\mathcal{K} g_{n+1}(z)
$$

and

$$
g_{n+1, \gamma}(z)=\xi_{n+1}+\mathcal{K}_{\gamma} g_{n+1, \gamma}(z)
$$

We also have

$$
g_{n+1} \circ \gamma(z)=\xi_{n+1}+\mathcal{K}_{\gamma}\left(g_{n+1} \circ \gamma(z)\right) .
$$

From $g_{n+1}$ (as well as $g_{n+1, \gamma}$ ), which is holomorphic outside $\Delta$ and continuous in $\mathbb{C}$, we get a holomorphic map $h_{i}$ (as well as $h_{n+1, \gamma}$ ) in $\Delta$ for $i=1, \cdots, n$. Then we form two holomorphic maps

$$
F_{n+1}=\left(h_{1}, \cdots, h_{n}, h_{n+1}\right): \Delta \rightarrow Y_{n+1}
$$

and

$$
F_{n+1, \gamma}=\left(h_{1} \circ \gamma, \cdots, h_{n} \circ \gamma, h_{n+1, \gamma}\right): \Delta \rightarrow Y_{n+1}
$$


Since $\Delta$ is simply connected and since $p_{n+1}: T\left(E_{n+1}\right) \rightarrow Y_{n+1}$ is the universal cover, we can lift $F_{n+1}$ and $F_{n+1, \gamma}$ to two holomorphic maps

$$
f_{\Delta, n+1}: \Delta \rightarrow T\left(E_{n+1}\right) \text { and } f_{\Delta, n+1, \gamma}: \Delta \rightarrow T\left(E_{n+1}\right)
$$

such that $p_{n+1} \circ f_{\Delta, n+1}=F_{n+1}$ and $p_{n+1} \circ f_{\Delta, n+1, \gamma}=F_{n+1, \gamma}$.

Under the assumption of the trivial monodromy, we know that $f_{\Delta, n}=f_{\Delta, n} \circ \gamma$ (see the proof of Theorem A). That is, $h_{i}=h_{i} \circ \gamma$ and $g_{i}=g_{i} \circ \gamma$ for all $1 \leq i \leq n$. Thus $\mathcal{K}_{\gamma}=\mathcal{K}$. Since the fixed point is unique, we get

$$
g_{n+1} \circ \gamma=g_{n+1, \gamma}=g_{n+1} .
$$

This implies that $h_{n+1} \circ \gamma=h_{n+1}$ and $F_{n+1} \circ \gamma=F_{n+1}$ and $f_{\Delta, n+1} \circ \gamma=f_{\Delta, n+1}$. Since this holds for all $\gamma \in \Gamma$, the map $f_{\Delta, n+1}$ defines a holomorphic map $f_{n+1}$ : $X \rightarrow T\left(E_{n+1}\right)$ such that $p \circ f_{n+1}=f_{n}$. Therefore, $f_{n+1}$ is a lift of $f_{n}$. This completes the proof.

Proof of Corollary 2.1. This follows at once from Theorem [C] Proposition 1.17. and Theorem $\mathrm{B}$.

\section{Proof of Theorem D}

Let $E$ be an arbitrary closed set in $\widehat{\mathbb{C}}$ such that 0,1 , and $\infty$ are in $E$, and let $X$ be a hyperbolic Riemann surface with a basepoint $x_{0}$.

We will need the following result; see Theorem $\mathrm{C}$ in 4 .

Theorem 6.1. Let $\phi: X \times E \rightarrow \widehat{\mathbb{C}}$ be a holomorphic motion. Suppose the restriction of $\phi$ to $X \times E^{\prime}$ extends to a holomorphic motion $\widetilde{\phi}: X \times \widehat{\mathbb{C}} \rightarrow \widehat{\mathbb{C}}$, whenever $\{0,1, \infty\} \subset E^{\prime} \subset E$ and $E^{\prime}$ is finite. Then $\phi$ can be extended to a holomorphic motion of $\widehat{\mathbb{C}}$ over $X$.

Proof of Theorem D, Let $\phi: X \times E \rightarrow \widehat{\mathbb{C}}$ be a holomorphic motion with the property that $\phi$ restricted to $X \times E^{\prime}$ has trivial monodromy, where $E^{\prime}$ is any finite subset of $E$, containing 0,1 , and $\infty$.

Fix some $E^{\prime} \subset E$ such that $E^{\prime}$ contains the points $0,1, \infty$ and $\phi$ restricted to $X \times E^{\prime}$ has trivial mondromy. Let $E_{0}=E^{\prime}$. Consider $E_{1}=E_{0} \cup\left\{\zeta_{1}\right\}$ for $\zeta_{1} \notin E_{0}$. Inductively, consider $E_{n+1}=E_{n} \cup\left\{\zeta_{n+1}\right\}$ for $\zeta_{n+1} \notin E_{n}$ for all $n \geq 0$. Then we eventually get a countable set $E_{\infty}=\bigcup_{n=0}^{\infty} E_{n}$ in $\widehat{\mathbb{C}}$. We can assume that $E_{\infty}$ is dense in $\widehat{\mathbb{C}}$.

Define $\phi_{0}:=\phi$ restricted to $X \times E_{0}$. Using Theorem C and Corollary 2.1 inductively, we know that the holomorphic motion $\phi_{n}: X \times E_{n} \rightarrow \widehat{\mathbb{C}}$ can be extended to a holomorphic motion $\phi_{n+1}: X \times E_{n+1} \rightarrow \widehat{\mathbb{C}}$ with trivial monodromy for all $n \geq 0$. Thus we can extend $\phi_{0}$ to a holomorphic motion $\phi_{\infty}: X \times E_{\infty} \rightarrow \widehat{\mathbb{C}}$. Since $E_{\infty}$ is dense in $\widehat{\mathbb{C}}$, it can be further extended to a holomorphic motion $\widetilde{\phi}: X \times \widehat{\mathbb{C}} \rightarrow \widehat{\mathbb{C}}$. The conclusion now follows from Theorem 6.1.

Remark 6.2. Let $G$ be a group of Möbius transformations, such that the closed set $E$ is $G$-invariant. Let $\phi: X \times E \rightarrow \widehat{\mathbb{C}}$ be a $G$-equivariant holomorphic motion; see Definition 1.3. If $\phi$ has the property that $\phi$ restricted to $X \times E^{\prime}$ has trivial monodromy, where $E^{\prime}$ is any subset of E, containing the points 0,1 , and $\infty$, then $\phi$ can be extended to a holomorphic motion $\widetilde{\phi}: X \times \widehat{\mathbb{C}} \rightarrow \widehat{\mathbb{C}}$ which is also $G$ equivariant. The proof is very similar to the proof of Theorem 1 in [10] or of Theorem B in [3]. 
Remark 6.3. The question of liftings of holomorphic maps into complex manifolds is a more general and fundamental question. In our paper, we have studied liftings of holomorphic maps into Teichmüller spaces and their intimate relationship with extending holomorphic motions. In future research, we plan to investigate whether the tools and techniques in our paper can be extended to other kinds of Teichmüller spaces.

\section{ACKNOWLEDGMENT}

We are extremely grateful to the referee for valuable suggestions and important comments.

\section{REFERENCES}

[1] Lars V. Ahlfors, Lectures on quasiconformal mappings, 2nd ed., University Lecture Series, vol. 38, American Mathematical Society, Providence, RI, 2006. MR2241787

[2] Lars Ahlfors and Lipman Bers, Riemann's mapping theorem for variable metrics, Ann. of Math. (2) 72 (1960), 385-404, DOI 10.2307/1970141. MR0115006

[3] Michael Beck, Yunping Jiang, and Sudeb Mitra, Normal families and holomorphic motions over infinite dimensional parameter spaces, Conformal dynamics and hyperbolic geometry, Contemp. Math., vol. 573, Amer. Math. Soc., Providence, RI, 2012, pp. 1-9, DOI 10.1090/conm/573/11409. MR2964070

[4] Michael Beck, Yunping Jiang, Sudeb Mitra, and Hiroshige Shiga, Extending holomorphic motions and monodromy, Ann. Acad. Sci. Fenn. Math. 37 (2012), no. 1, 53-67, DOI 10.5186/aasfm.2012.3713. MR2920423

[5] Lipman Bers and H. L. Royden, Holomorphic families of injections, Acta Math. 157 (1986), no. 3-4, 259-286, DOI 10.1007/BF02392595. MR857675

[6] E. M. Chirka, On the extension of holomorphic motions, Doklady Mathematics, 70, No. 1, (2004), 516-519.

[7] Adrien Douady and Clifford J. Earle, Conformally natural extension of homeomorphisms of the circle, Acta Math. 157 (1986), no. 1-2, 23-48, DOI 10.1007/BF02392590. MR857678

[8] Clifford J. Earle, On holomorphic cross-sections in Teichmüller spaces, Duke Math. J. 36 (1969), 409-415. MR0254233

[9] Clifford J. Earle and Irwin Kra, On holomorphic mappings between Teichmüller spaces, Contributions to analysis (a collection of papers dedicated to Lipman Bers), Academic Press, New York, 1974, pp. 107-124. MR0430319

[10] C. J. Earle, I. Kra, and S. L. Krushkal', Holomorphic motions and Teichmüller spaces, Trans. Amer. Math. Soc. 343 (1994), no. 2, 927-948, DOI 10.2307/2154750. MR1214783

[11] Clifford J. Earle and Sudeb Mitra, Variation of moduli under holomorphic motions, In the tradition of Ahlfors and Bers (Stony Brook, NY, 1998), Contemp. Math., vol. 256, Amer. Math. Soc., Providence, RI, 2000, pp. 39-67, DOI 10.1090/conm/256/03996. MR.1759669

[12] Frederick P. Gardiner, Yunping Jiang, and Zhe Wang, Holomorphic motions and related topics, Geometry of Riemann surfaces, London Math. Soc. Lecture Note Ser., vol. 368, Cambridge Univ. Press, Cambridge, 2010, pp. 156-193. MR2665009

[13] John Hamal Hubbard, Sur les sections analytiques de la courbe universelle de Teichmüller, Mem. Amer. Math. Soc. 4 (1976), no. 166, ix+137, DOI 10.1090/memo/0166. MR0430321

[14] John Hamal Hubbard, Teichmüller theory and applications to geometry, topology, and dynamics. Vol. 1, Matrix Editions, Ithaca, NY, 2006. MR2245223

[15] Yunping Jiang and Sudeb Mitra, Some applications of universal holomorphic motions, Kodai Math. J. 30 (2007), no. 1, 85-96, DOI 10.2996/kmj/1175287624. MR2319079

[16] Yunping Jiang and Sudeb Mitra, Douady-Earle section, holomorphic motions, and some applications, Quasiconformal mappings, Riemann surfaces, and Teichmüller spaces, Contemp. Math., vol. 575, Amer. Math. Soc., Providence, RI, 2012, pp. 219-251, DOI 10.1090/conm/575/11400. MR2933902

[17] Yunping Jiang, Sudeb Mitra, and Zhe Wang, Liftings of holomorphic maps into Teichmüller spaces, Kodai Math. J. 32 (2009), no. 3, 547-563, DOI 10.2996/kmj/1257948895. MR2582017 
[18] G. S. Lieb, Holomorphic motions and Teichmüller space, Ph.D. dissertation, Cornell University, 1990.

[19] R. Mañé, P. Sad, and D. Sullivan, On the dynamics of rational maps, Ann. Sci. École Norm. Sup. (4) 16 (1983), no. 2, 193-217. MR732343

[20] Sudeb Mitra, Teichmüller spaces and holomorphic motions, J. Anal. Math. 81 (2000), 1-33, DOI 10.1007/BF02788984. MR.1785276

[21] Sudeb Mitra, Extensions of holomorphic motions, Israel J. Math. 159 (2007), 277-288, DOI 10.1007/s11856-007-0047-1. MR2342482

[22] Subhashis Nag, The Torelli spaces of punctured tori and spheres, Duke Math. J. 48 (1981), no. 2, 359-388. MR620255

[23] Subhashis Nag, The complex analytic theory of Teichmüller spaces, Canadian Mathematical Society Series of Monographs and Advanced Texts, John Wiley \& Sons, Inc., New York, 1988. A Wiley-Interscience Publication. MR927291

[24] H. Shiga, Extension of holomorphic motions and monodromy, preprint.

[25] Zbigniew Slodkowski, Holomorphic motions and polynomial hulls, Proc. Amer. Math. Soc. 111 (1991), no. 2, 347-355, DOI 10.2307/2048323. MR.1037218

[26] Dennis P. Sullivan and William P. Thurston, Extending holomorphic motions, Acta Math. 157 (1986), no. 3-4, 243-257, DOI 10.1007/BF02392594. MR857674

[27] Eberhard Zeidler, Nonlinear functional analysis and its applications. I, Springer-Verlag, New York, 1986. Fixed-point theorems; Translated from the German by Peter R. Wadsack. MR.816732

Department of Mathematics, Queens College of the City University of New York, New York; and Department of Mathematics, The Graduate Center, CUNy, New York, NEW YORK

Email address: yunping.jiang@qc.cuny.edu

Department of Mathematics, Queens College of the City University of New York, New York; and Department of Mathematics, The Graduate Center, CUnY, New York, NEW YORK

Email address: sudeb.mitra@qc.cuny.edu 